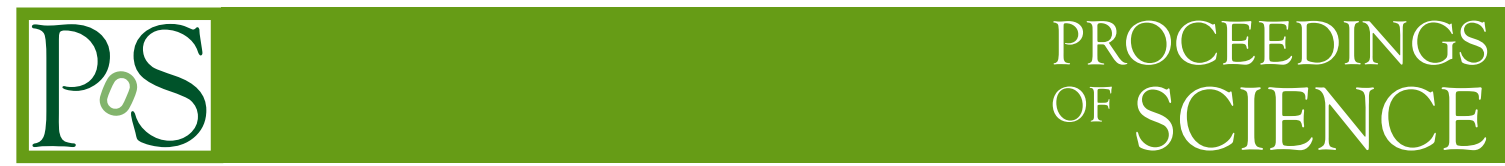

\title{
Weak gravitational lensing
}

\author{
Alan Heavens* \\ Imperial College London \\ E-mail: a.heavens@imperial.ac.uk
}

Weak gravitational lensing by inhomogeneities along the line-of-sight alters the shapes, sizes and fluxes of distant sources such as galaxies, and distorts the pattern of continuous fields such as the microwave background radiation. In this talk I will review this relatively young scientific field, with particular emphasis on its power for studying dark energy and modified gravity. I will discuss the challenges it faces, summarize results from recent survey analyses, and finally consider the prospects for the future

Frontiers of Fundamental Physics 14 - FFP14,

15-18 July 2014

Aix Marseille University (AMU) Saint-Charles Campus, Marseille

\footnotetext{
* Speaker.
} 